\title{
1201 - Liquid Cell TEM Imaging Techniques and Optimization for Biological Samples
}

Madeline Dukes ${ }^{1}$, Deborah Kelly ${ }^{2}$ and Cameron Varano ${ }^{2}$

${ }^{1}$ Protochips, Inc., Cross Hill, South Carolina, United States, ${ }^{2}$ Pennsylvania State University, University Park, Pennsylvania, United States

Utilization of state-of-the-art in situ and operando liquid-cell transmission electron microscopy (LCTEM) techniques has expanded exponentially, permitting scientists to observe dynamic processes occurring in liquid at the nanoscale. Dedicated LC-TEM sample holders enable direct imaging of hydrated and liquid samples using a standard transmission electron microscope (TEM). This environment permits the samples to move and react freely, enabling real-time processes and fine movements to be observed at a resolution of a few nanometers, leading to a more complete understanding of the dynamics of individual systems that static snapshots cannot fully convey [1].

LC-TEM experiments are performed by encapsulating the samples between microchips containing electron transparent membranes which are secured in dedicated sample holders (Figure 1). Microfluidic tubing integrated into the holder enables liquid to be delivered via flow to the sample chamber during the experiment and allows the liquid environment to be maintained or adjusted during the course of the experiment.

Although LC-TEM has enjoyed wide acceptance in materials applications, challenges related to both sample preparation and imaging of beam sensitive samples in liquid have resulted in the biological community being slower to adopt LC-TEM techniques. This tutorial will explore the advantages that LCTEM can offer life science applications, how to load and assemble in-situ liquid TEM holders, and how key challenges related to the technique can be minimized to facilitate successful LC-TEM results. Practical strategies covered will include experimental planning and design for LC-TEM, optimizing sample preparation workflows, techniques to improve TEM imaging quality in liquid, such as the use of heavy metal staining to increase the contrast (Figure 2) [2, 3], and mitigation of radiolytic beam damage. These and other topics will be discussed with the goal of enabling researchers to be successful when working with biological samples in situ. In summary, this tutorial will highlight the utility of LCTEM for biological applications and provide an introduction to techniques to improve in situ LC-TEM results for biological samples.
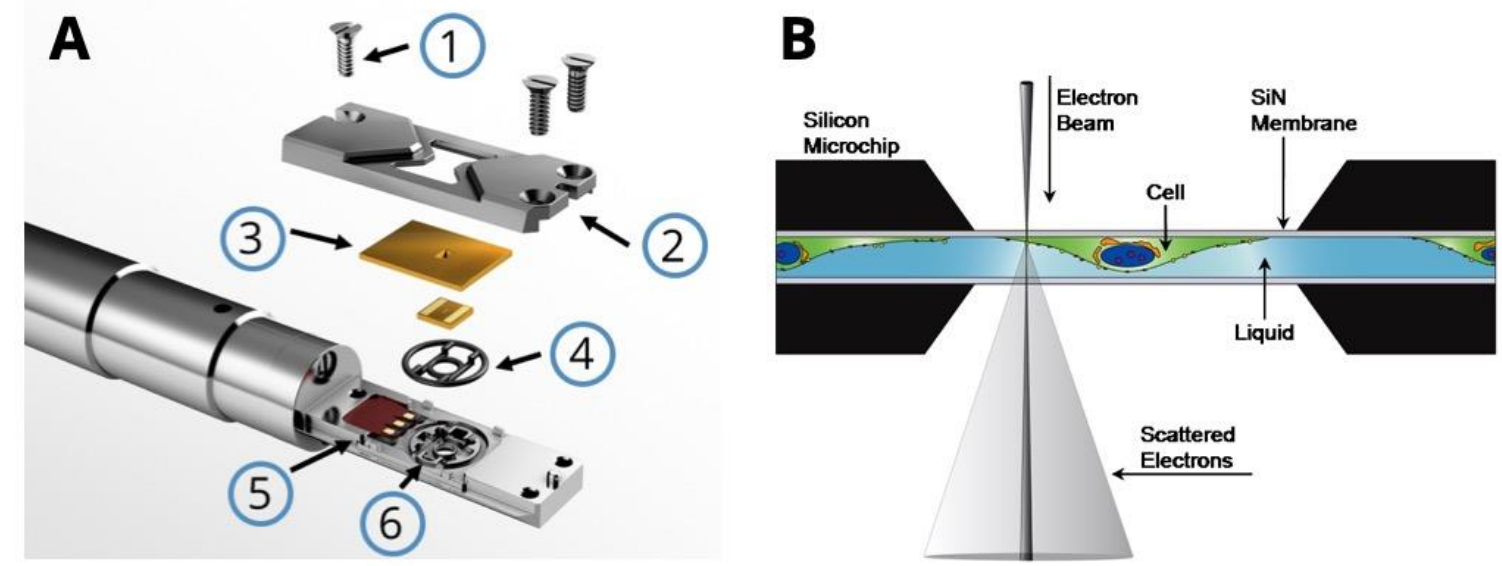
Figure 1. (A) Exploded view of the tip of a Protochips' Poseidon Select LC-TEM Holder. (1) Screws for securing the tip. (2) The lid design maximizes EDS signal (3) Each experiment uses a pair of E-chips that support a SiN membrane. (4) Gasket creates a hermetic seal (5) Integrated electrodes for heating and electrochemistry. (6) Microfluidic tubing enables continuous liquid flow. (B) Cross section of a eukaryotic cell contained in the sample chamber of an LC-TEM holder.
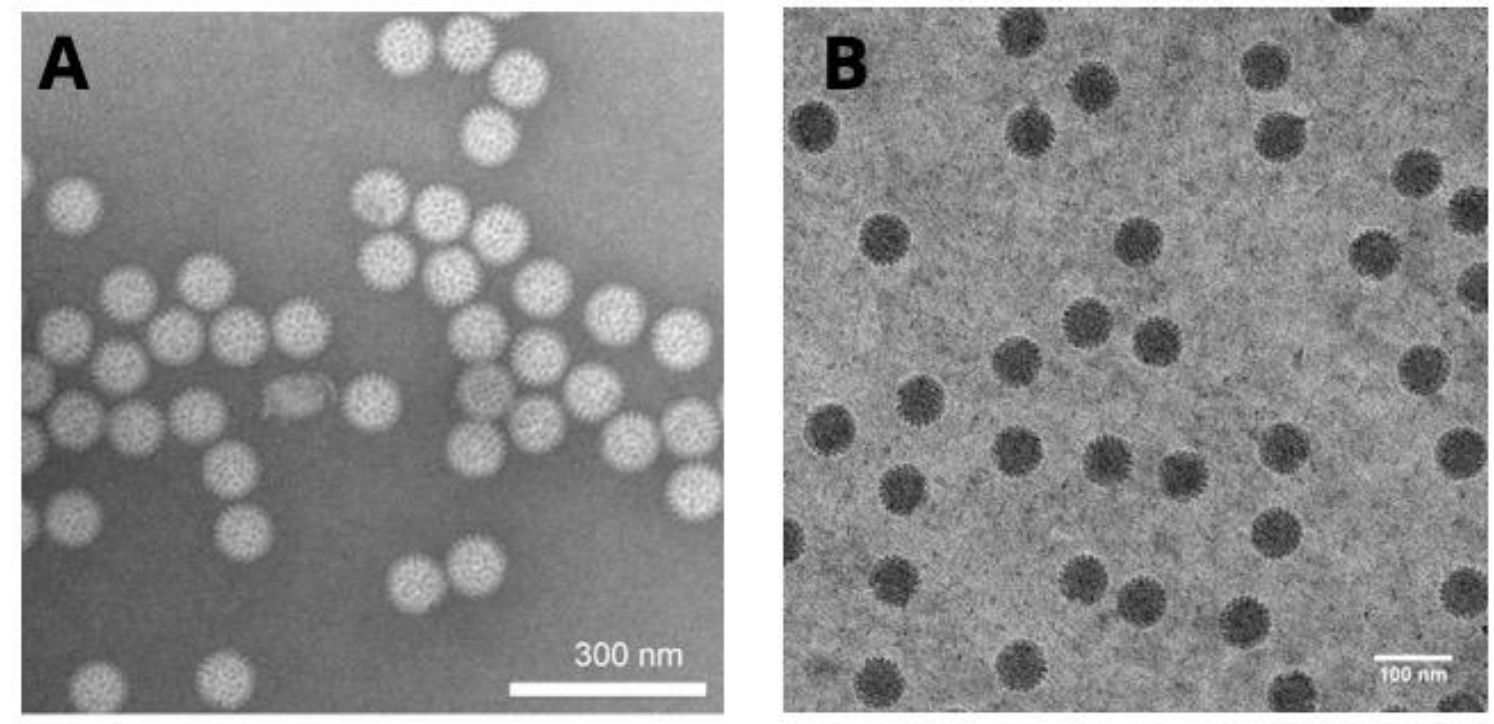

Figure 2. Double layer rotavirus imaged in liquid using LC-TEM (A) with uranyl formate staining (B) without staining.

\section{References}

[1] Demmert et al., in (2016) 'Visualizing Macromolecules in Liquid at the Nanoscale', in Ross, F.M. (ed.) Liquid Cell Electron Microscopy. Cambridge: Cambridge University Press, pp. 334-355.

[2] Gilmore et al., Lab on a Chip, 13.2 (2013): 216-219

[3] Dukes et al., Microscopy and Microanalysis, 20.2 (2014): 338-345 